University of Nebraska - Lincoln DigitalCommons@University of Nebraska - Lincoln

Faculty Publications from the Department of

Electrical and Computer Engineering

Electrical \& Computer Engineering, Department of

2011

\title{
Substrate wobble compensation for in situ spectroscopic ellipsometry measurements
}

Blaine Johs

J.A. Woollam Co., Inc.

Ping He

J.A. Woollam Co.

Follow this and additional works at: http://digitalcommons.unl.edu/electricalengineeringfacpub

Johs, Blaine and He, Ping, "Substrate wobble compensation for in situ spectroscopic ellipsometry measurements" (2011). Faculty

Publications from the Department of Electrical and Computer Engineering. 226.

http://digitalcommons.unl.edu/electricalengineeringfacpub/226

This Article is brought to you for free and open access by the Electrical \& Computer Engineering, Department of at DigitalCommons@University of Nebraska - Lincoln. It has been accepted for inclusion in Faculty Publications from the Department of Electrical and Computer Engineering by an authorized administrator of DigitalCommons@University of Nebraska - Lincoln. 


\title{
Substrate wobble compensation for in situ spectroscopic ellipsometry measurements
}

\author{
Blaine Johs ${ }^{\mathrm{a})}$ and Ping $\mathrm{He}$ \\ J.A. Woollam Co., Inc., 645 M St., \#102, Lincoln, Nebraska 68508
}

(Received 27 October 2010; accepted 17 January 2011; published 14 February 2011)

\begin{abstract}
Spectroscopic ellipsometry (SE) is an optical characterization technique that has been implemented on molecular beam epitaxy chambers for in situ characterization and growth control. SE measurements require collecting light reflected obliquely from the substrate. As the substrate is usually rotating during growth (to promote uniformity), acquiring accurate in situ SE data typically requires a substrate manipulator with low wobble (preferably $<0.1^{\circ}$ ). To overcome the low wobble manipulator requirement, the authors have designed and tested a return path SE configuration that compensates for the effects of substrate wobble. The prototype wobble compensation system demonstrated the near elimination of beam precession in the outgoing ellipsometer beam. The accuracy of the SE data acquired in the return path configuration during substrate rotation was also studied. (C) 2011 American Vacuum Society. [DOI: 10.1116/1.3555332]
\end{abstract}

\section{INTRODUCTION}

Spectroscopic ellipsometry (SE) is a powerful optical characterization technique ${ }^{1,2}$ that has been implemented on molecular beam epitaxy (MBE) chambers for in situ characterization and real time control of substrate temperature, layer thickness, and composition. ${ }^{3-6}$ SE measurements require collecting light reflected from the substrate, which in a MBE chamber is usually rotated to promote uniformity. If the substrate surface is not exactly perpendicular to the axis of rotation, the substrate will "wobble" during rotation, and the reflected SE beam will precess as the substrate rotates. Acquiring accurate and precise in situ SE data requires a substrate manipulator with low wobble (preferably $<0.1^{\circ}$ ), which is challenging to design and maintain when operating in UHV and at high temperatures. While a novel ultrastable manipulator capable of "dialing out" wobble by externally controlling the substrate tilt has been demonstrated, ${ }^{7}$ this technology is expensive. Most in situ SE measurements on MBE chambers have been performed by using a large measurement beam to overfill the detector aperture and adjusting the manipulator or substrate mounting to keep the beam precession small enough so that some part of the large beam is always collected by the detector during rotation. ${ }^{8}$ Despite these workarounds, substrate wobble issues have limited the general applicability of in situ SE for many MBE applications.

As suggested by Haberland et al., ${ }^{9}$ a return path ellipsometer configuration with a spherical mirror and beamsplitter can compensate for the effects of substrate wobble. We have implemented a return path ellipsometer design which eliminates the beamsplitter by incorporating novel prism beam folding optics to provide minimal spacing between the incoming and outgoing ellipsometer beams (which in turn reduces the spacing of the beams on the substrate). A prototype system was constructed and tested on an ex situ "wobble

${ }^{a)}$ Electronic mail: bjohs@jawoollam.com simulator" to demonstrate the near elimination of beam precession in the outgoing beam. The impact of variations in the beam angle and plane of incidence (with respect to the rotating sample surface) on the accuracy of the SE data was also studied.

\section{EXPERIMENT}

\section{A. Return path configurations}

To compensate for the effects of substrate wobble, Haberland et al. suggested a return path ellipsometer configuration in which a spherical mirror reflects the beam back to the sample, returning the beam on the same path as the incoming beam, independent of the substrate tilt. ${ }^{9}$ This clever configuration is based on the reflecting properties of a spherical mirror: a beam initiated at the focal point of a spherical mirror will be reflected back to the exact same point, regardless of the beam direction (as long as the beam hits the spherical mirror, of course). Figure 1(a) shows a schematic of this return path configuration. The main benefit of this configuration is that the outgoing and return beams hit the sample at the same location. However, a beamsplitter is required to implement this configuration, which makes this configuration impractical for a wide spectral range (e.g., 250-1000 nm) SE system: nonabsorbing achromatic beamsplitters are not available, and in the best case (a perfect 50/50 beamsplitter) the light throughput would be reduced by a factor of 4 . Furthermore, the beamsplitter can induce polarization effects in the beams that need to be corrected for accurate SE data.

Figures 1(b) and 1(c) show alternative return path configurations proposed in this work. These configurations replace the beamsplitter with novel prism-based beam folding optics (J.A. Woollam Co., Inc., patent pending): the beam is reflected from two right angle prisms which are oriented with their plane of incidences rotated $90^{\circ}$. This geometry cancels the reflection-induced polarization state changes of each individual prism, providing a $90^{\circ}$ turn in the beam direction with essentially no net change in the polarization state of the 
a)
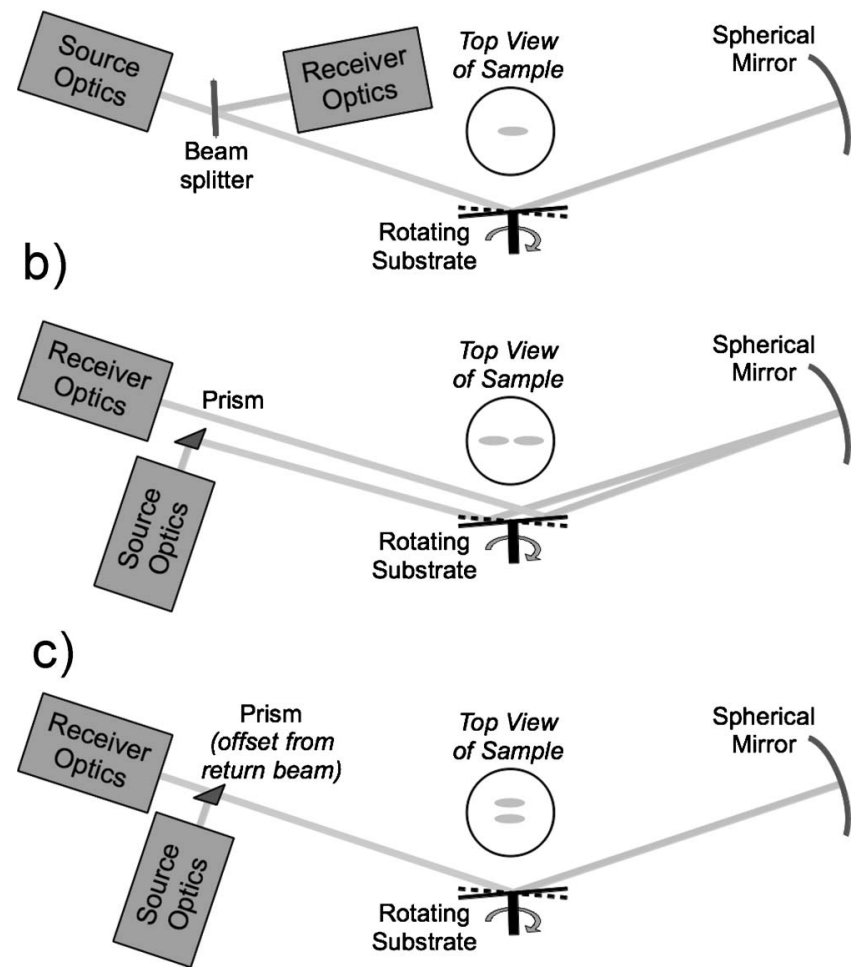

FIG. 1. Return path ellipsometer configurations: (a) beamsplitter configuration suggested by Haberland et al., (b) prism folding optics with beam offset in the angle of incidence, and (c) prism folding optics with beam offset in the plane of incidence.

beam. Folding the beam $90^{\circ}$ allows the incoming and outgoing beams to be positioned close together $(<10 \mathrm{~mm}$ spacing at the folding optics) even though the SE source and receiver optics occupy a relatively large volume (typically 100 $\times 100 \times 100 \mathrm{~mm}^{3}$ or larger). This also allows the source and receiver optics to be position together on one side of the chamber, with a much smaller footprint requirement for the spherical return mirror on the opposite side of the chamber. The downside to these alternative configurations is that the incoming and outgoing beams hit at different locations on the sample. The easier configuration to visualize is shown in Fig. 1(b), in which the beams are offset in the angle of incidence. In this case, the two beams are positioned "end to end" on the sample (as shown in the "top view" in the figure). In Fig. 1(c) the beams are offset in the plane of incidence; this is harder to visualize from the "side view," but the top view in the figure illustrates that beams are positioned "side by side" on the sample in this configuration. This is the preferred configuration, as it minimizes the footprint of the two beams on the sample, and both reflections on the sample have the same angle of incidence. Assuming the path length from the optics to the substrate is the same as the path length from the substrate to the spherical return mirror, the spacing of the beams on the sample will be one half the separation distance at the folding prism optics.

\section{B. Ex situ prototype testing}

A prototype system was constructed to demonstrate the effectiveness of the return path wobble compensation SE concept. It was tested ex situ on an optical bench (nominal angle of incidence $=75^{\circ}$ ) using a wobble simulator consisting of a tilt stage with a 4 in. native oxide $\mathrm{Si}$ wafer, mounted on a stepper motor shaft. The path length from the optics to the sample and from the sample to the spherical mirror was set to $\approx 1 \mathrm{~m}$. Therefore, a spherical mirror with a $1 \mathrm{~m}$ radius of curvature $R$ was chosen (which corresponds to a mirror focal length $F$ of $0.5 \mathrm{~m}$ as $R=2 F$ ). The tilt stage on the wobble simulator was adjusted such that the beam precession (peakto-peak translation of the beam) at the spherical mirror was $\approx 28 \mathrm{~mm}$, which over the $1 \mathrm{~m}$ path length corresponds to an angular wobble of $\pm 0.8^{\circ}$. The source optics were adjusted to focus the beam on to the sample, resulting in elliptical beam spot size on the sample of $\approx 3 \times 11 \mathrm{~mm}$. The spacing between the two reflections on the sample was $\approx 10 \mathrm{~mm}$, due to the relatively large spacing $(\approx 20 \mathrm{~mm})$ between the incoming and outgoing beams in the prototype beam folding optics.

The tilt of the spherical return mirror was adjusted such that the return beam was centered on the receiver optics aperture, and the mirror was focused to minimize the beam precession at the receiver optics aperture while the substrate was rotating. The observed beam precession was $\approx 1 \mathrm{~mm}$ at the receiver, effectively demonstrating a $>20 \times$ reduction in beam precession. The residual beam precession is due to variations in the substrate "height" during rotation; while the return path configuration corrects for angular variation of the substrate during rotation, it does not correct for the small height variations due to the ellipsometer beams hitting the substrate slightly off center. Since the beam size at the receiver aperture was $\approx 10 \mathrm{~mm}, \mathrm{a} \approx 1 \mathrm{~mm}$ beam precession is completely acceptable.

A dual rotating compensator SE system was used for the prototype testing (Model RC2, J.A. Woollam Co., Inc.). In addition to the traditional ellipsometric measurements of $\Psi$ and $\Delta$, the dual rotating compensator configuration ${ }^{10}$ enables simultaneous collection of all 16 Mueller matrix (MM) elements, ${ }^{2}$ which can fully characterize samples which exhibit an anisotropic and/or depolarizing optical response. While the Mueller matrix measurement capability is not required for most in situ characterization applications (as most samples are effectively isotropic), it was useful for studying plane of incidence variations, which are discussed in Sec. II C.

To acquire accurate and precise SE data, stability in the collected beam intensity is paramount. Figure 2 plots the average signal intensity collected by the SE detector during substrate rotation. For this test, a slow $\approx 90 \mathrm{~s}$ substrate rotation period was used, with a fast SE acquisition time of $0.8 \mathrm{~s}$. From Fig. 2, the calculated standard deviation in signal intensity during substrate rotation was less than $2 \%$. Note that without the return path configuration, no portion of the beam would be collected during much of the rotation period, resulting in a $100 \%$ signal intensity variation. 


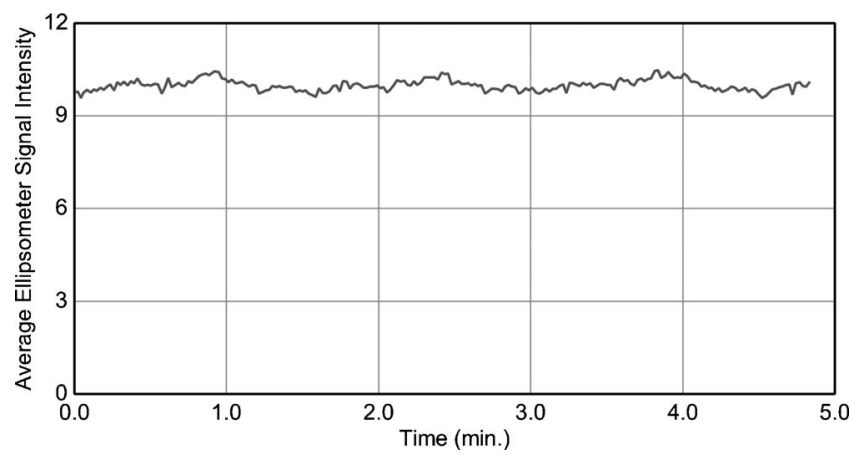

FIG. 2. Variation in average SE signal intensity during substrate rotation.

\section{SE: Data accuracy}

While Sec. II B demonstrated that the return path configuration was effective in minimizing the beam precession and signal intensity variation observed during substrate rotation, the ellipsometer beam is still subject to rotation induced changes in the angle of incidence and plane of incidence, both of which are defined with respect to the substrate surface. The effects of these changes on the SE data are discussed in this section.

The polarized optical response of the return path ellipsometer configuration can be calculated by the Mueller matrices $^{2}$ shown in Fig. 3. Note that the MM for the substrate reflections (an isotropic sample matrix) has zero elements in the off-diagonal $2 \times 2$ blocks. The nonzero Mueller matrix elements are denoted $N, C$, and $S$, which are defined in terms of the traditional ellipsometric parameters: $N=\cos (2 \Psi), C$ $=\sin (2 \Psi) \cos (\Delta)$, and $S=\sin (2 \Psi) \sin (\Delta)$. The NCS parameters can be calculated by an optical model for the sample and are functions of the angle of incidence, wavelength of light, layer thicknesses, and optical constants of the substrate and layers. The rotation matrices (the first and last matrices in the matrix equation in Fig. 3) account for changes in the plane of incidence with respect to the azimuthal orientations of the optics. Multiplying by a rotation matrix interchanges values between the inner two rows and columns of the matrix which is operated on. For example, if an isotropic sample Mueller matrix is rotated, a fraction of the $m_{33}$ element [which corresponds to $C=\sin (2 \Psi) \cos (\Delta)$ for an isotropic sample] can appear in the $m_{23}$ and $m_{32}$ elements, which are zero for an isotropic sample. As the beam reflects from the spherical return mirror at essentially normal incidence, the corresponding Mueller matrix simply applies a $180^{\circ}$ phase reversal.

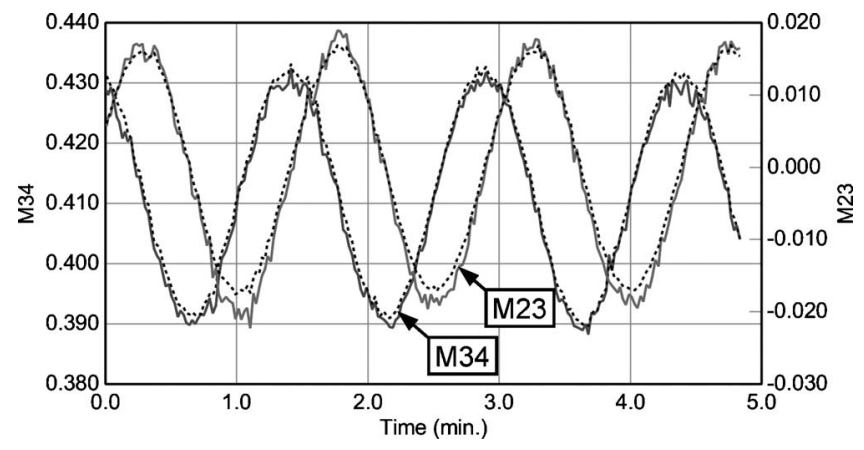

FIG. 4. Mueller matrix data acquired during substrate rotation: $m_{23}$ is sensitive to plane of incidence variations; $m_{34}$ is sensitive to angle of incidence variations. The dashed curves are calculated from the best fit optical model.

To study rotation induced changes in the angle and plane of incidence, Mueller matrix data were acquired ( $0.8 \mathrm{~s}$ acquisition period) while the substrate was slowly rotated $(90 \mathrm{~s}$ rotation period). Selected Mueller matrix elements from this experiment are plotted in Fig. 4: the $m_{23}$ data are sensitive to changes in the plane of incidence (due to rotation effects described in the preceding paragraph), while the $m_{34}$ data [which correspond to $S=\sin (2 \Psi) \sin (\Delta)$ ] are sensitive to changes in the angle of incidence. As expected, the curves oscillate with the substrate rotation period and are $90^{\circ}$ out of phase from each other. The black dashed curves in Fig. 4 are calculated from the best fit optical model (the fit included all the measured Mueller matrix elements in the analysis). The fit parameters were the angle of incidence and the source and receiver azimuthal rotation angles (the native oxide was fixed at the previously determined value). Figure 5 plots these fit parameters versus time, which also oscillate with the substrate rotation period. The two rotation parameter curves lie essentially on top of each other and are $90^{\circ}$ out of phase with the angle of incidence. Note that the variation in these angles is $\pm 0.4^{\circ}$, which is half the angular variation estimated from the beam precession measurement. This is as expected as a beam incident on a sample with angle $\theta$ is reflected at an angle of $2 \theta$ with respect to the incident beam.

If the SE data acquisition period is less than the substrate rotation period, the rotation induced variations in the data complicate the analysis of the SE data: either the angle of incidence and azimuthal rotation parameters must be fit for each spectra (which could correlate with other model fit parameters) or the angle and rotation parameters must be determined by a beam alignment sensor and fed into the analysis (though a beam alignment sensor on the outgoing beam

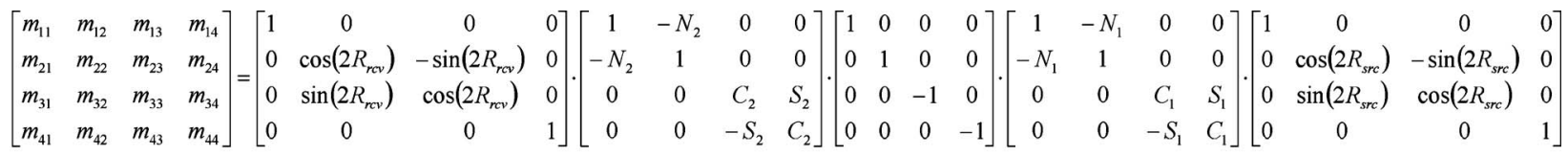

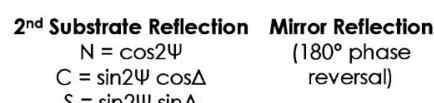

$$
\begin{aligned}
& \begin{array}{c}
N=\cos 2 \psi \\
C=\sin 2 \Psi \cos \Delta
\end{array}
\end{aligned}
$$
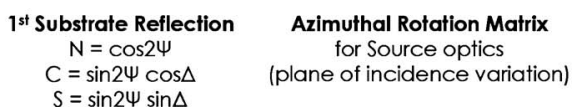

FIG. 3. Mueller matrices representing the return path ellipsometer configuration. 


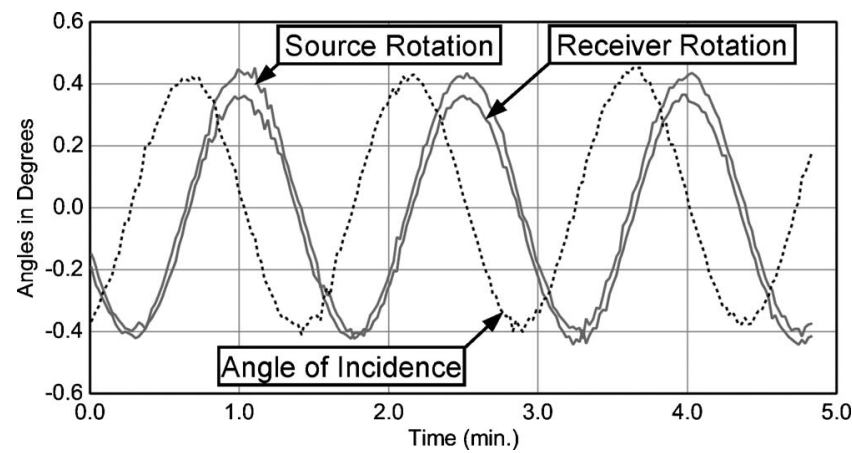

FIG. 5. Fit parameters derived by analyzing the data shown in Fig. 4: the angle of incidence is the dashed line; the source and receiver azimuthal rotation angles are the solid curves.

would have essentially no sensitivity due to the angular wobble compensation of the return path configuration). A straightforward solution to this problem is to synchronize the SE data acquisition period with the substrate rotation period $;^{7}$ this allows each SE data acquisition to average over the same range of angle and plane of incidence variations.

To visualize how the data can be averaged over the substrate rotation, consider the data plotted in Fig. 6: the $m_{34}$ data acquired at the maximum angle of incidence excursions appear symmetrically above and below the fast rotation "angle averaged" data, while the $m_{34}$ data at the maximum

a)

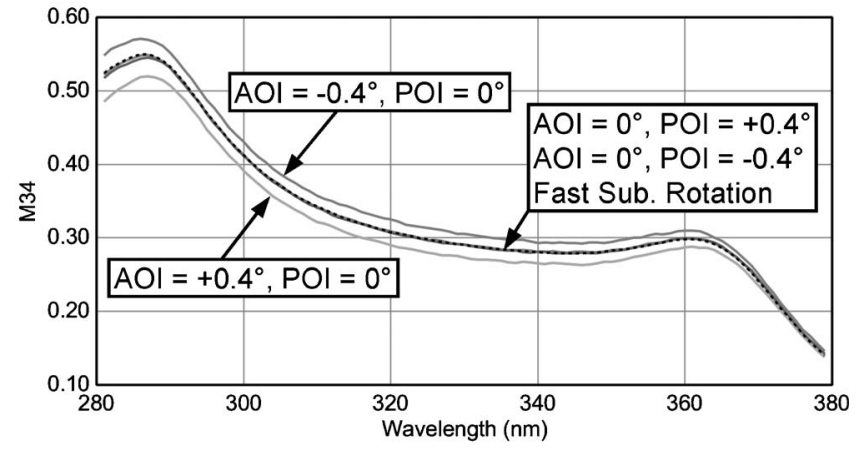

b)

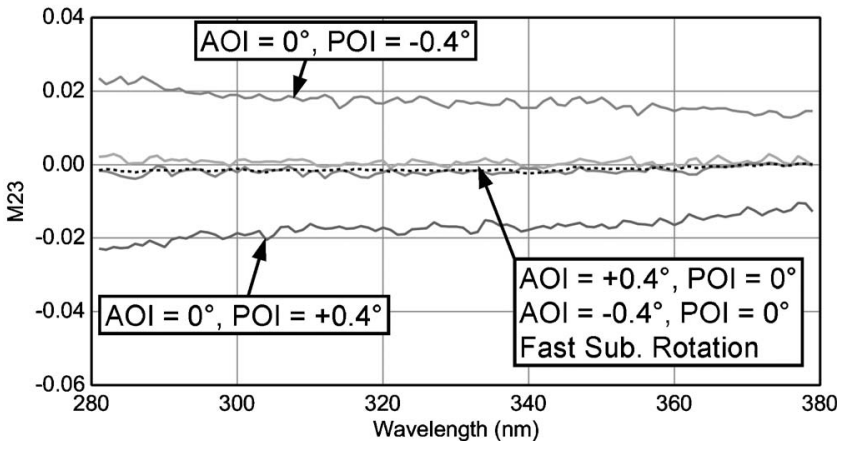

FIG. 6. Selected Mueller matrix spectra acquired during substrate rotation: the solid curves were acquired at the maximum angle and plane of incidence excursions with a slow substrate rotation, while the dashed curves were acquired with fast substrate rotation, synced to the SE data acquisition period to average over all substrate rotation angles. a)

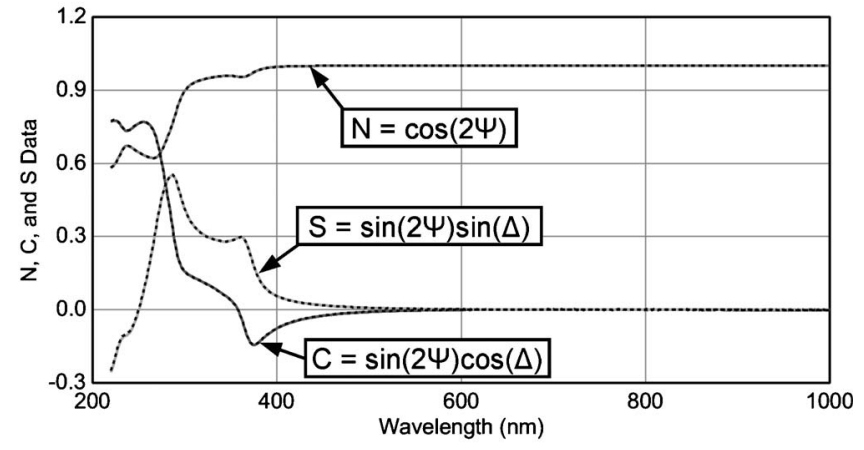

b)

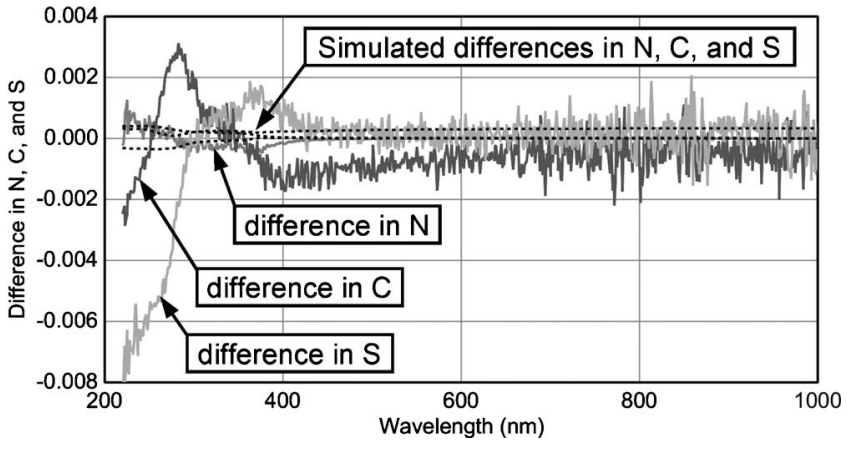

FIG. 7. SE. Data compared with and without substrate rotation: (a) on this scale, the solid curves (data with rotation) are essentially identical to the data without rotation (dashed curves); (b) the solid curves are the differences between the curves shown in (a), and the dashed curves are the model simulated differences due to rotation.

plane of incidence excursions are essentially identical to the fast rotation data. Likewise, the $m_{23}$ data at the maximum plane of incidence excursions appear symmetrically above and below the fast rotation angle averaged data, while the $m_{23}$ data at the maximum angle of incidence excursions are essentially identical to the fast rotation data. From these data it is clear that at least to the first order, accurate data can obtained by averaging over the substrate rotation period.

The definitive test of accuracy is a comparison of SE data acquired during substrate rotation (with the large $\pm 0.8^{\circ}$ wobble) with data acquired on a stationary aligned substrate, which is shown in Fig. 7. On the scale of Fig. 7(a), the SE data acquired with and without substrate rotation are essentially identical. However, a difference plot of the data sets [Fig. 7(b)] exposes significant differences. The simulated data differences (which are nonzero due to the slightly nonlinear response of $N, C$, and $S$ vs angle of incidence) are shown in Fig. 7(b) as dashed black lines and are much smaller than the experimentally measured values. Possible explanations for this discrepancy are sample nonuniformity (the stationary measurement sampled a different part of the substrate than the rotation-averaged measurement) and system misalignment (the two beams on the substrate were not exactly side by side). As shown in Fig. 7(a), the accuracy of SE data acquired in the return path configuration on a wob- 
bling substrate is quite good, but further work is required to experimentally determine the level of accuracy achievable with this approach.

\section{CONCLUSIONS}

Testing on an ex situ optical bench demonstrated that the return path SE configuration was highly effective in reducing the beam precession from $\approx 28$ to $\approx 1 \mathrm{~mm}$ for a $\pm 0.8^{\circ} \mathrm{sub}$ strate wobble and $1 \mathrm{~m}$ optics to sample path length, and the signal intensity variation was also reduced from $100 \%$ to $<2 \%$. Good agreement was observed between SE data acquired with and without substrate rotation, though further experiments will be performed to fully quantify the data accuracy of the return path configuration. While there may be some tradeoffs (reduced signal intensity due to two reflections from the sample and the two beams sample a larger area on the substrate), the return path configuration should provide significantly improved in situ SE performance and reduce the substrate manipulator wobble requirements, thereby increasing the suitability of SE for in situ MBE diagnostics.

${ }^{1}$ H. Fujiwara, Spectroscopic Ellipsometry, Principles and Applications (Wiley, New York, 2007).

${ }^{2}$ Handbook of Ellipsometry, edited by H. G. Tompkins and E. A. Irene (William Andrew, Norwich, NY, 2005).

${ }^{3}$ C. Herzinger, B. Johs, P. Chow, D. Reich, G. Carpenter, D. Croswell, and J. Van Hove, Mater. Res. Soc. Symp. Proc. 406, 347 (1996).

${ }^{4}$ E. Grassi, S. R. Johnson, M. Beaudoin, and K. S. Tsakalis, J. Vac. Sci. Technol. B 17, 1223 (1999).

${ }^{5}$ J. A. Roth, W. S. Williamson, D. H. Chow, G. L. Olson, and B. Johs, J. Vac. Sci. Technol. B 18, 1439 (2000).

${ }^{6}$ J. Phillips, D. Edwall, D. Lee, and J. Arias, J. Vac. Sci. Technol. B 19, 1580 (2001).

${ }^{7}$ G. N. Maracas, C. H. Kuo, S. Anand, R. Droopad, G. R. L. Sohie, and T. Levola, J. Vac. Sci. Technol. A 13, 727 (1995).

${ }^{8}$ B. Johs et al., Thin Solid Films 313-314, 490 (1998).

${ }^{9}$ K. Haberland, O. Hunderi, M. Pristovsek, J.-T. Zettler, and W. Richter, Thin Solid Films 313-314, 620 (1998).

${ }^{10}$ J. Lee, J. Koh, and R. W. Collins, Meas. Sci. Technol. 72, 1742 (2001). 